\title{
QUICK DETERMINATION OF CRYSTAL SIZE DISTRIBUTIONS OF ROCKS BY MEANS OF A COLOR SCANNER
}

\author{
SIMONE TARQUINI $^{1}$ AND PIETRO ARMIENTI ${ }^{2}$ \\ ${ }^{1}$ Istituto di Geocienze e Georisorse CNR, Via Moruzzi 1, 56100 Pisa Italy, ${ }^{2}$ Dipartimento di Scienze della \\ Terra, Università di Pisa, via S. Maria 5356100 Pisa Italy \\ e-mail: tarquini@igg.cnr.it, armienti@dst.unipi.it \\ (Accepted. February 1, 2003)
}

\begin{abstract}
An acquisition and analysis method based on a commercial, low-cost, high-resolution film scanner is presented. It allows to collect data from standard rock thin sections with a resolution up to $9.4 \mu \mathrm{m}$ per pixel. Common and general purpose facilities (scanner $+\mathrm{PC}+$ image analysis software) may thus be transformed in an appropriate tool for quantitative textural analysis of rocks. The procedure implies the acquisition of four images with crossed polarizers and one parallel light image. Crystal boundaries are extracted from fields in crossed polarizers, while markers for mineral recognition are obtained thresholding the parallel light image. The method is tested for fresh rocks with simple mineralogy (harzburgites and marbles) with no more than three phases, all exhibiting well distinct optical properties. Image processing is performed developing procedures with VISILOG 5.2 package. 2-D size data from binary images are converted to 3-D size data applying stereological corrections. 3-D data are reported in bi-logarithmic diagrams, plotting the crystal number density versus characteristic lengths. The harzburgite samples show a scale invariance of size distributions of olivine while mosaic equant marbles exhibit a different size distribution pattern, without scale invariance and a relative maximum.
\end{abstract}

Keywords: crystal size distribution, film scanner, petrology, rock texture.

\section{INTRODUCTION}

Several investigators have applied image analysis to solve petrological problems. Example applications relate to modal and grain-size analysis for rock classification or "liberation analysis" for optimal grinding of ore minerals (Petruck, 1988). The most varied image acquisition systems may be used: Scanning Electron Microscopes (SEM), Electronic Microprobes (through X-Ray elemental mapping), Transmitted Electron Microprobes (TEM), and petrographic microscope. A review of literature shows that until recently, only few image analysis tools have been developed in the field of textural and grain size analysis of thin sections in transmitted light microscopy. This is quite likely due to the great complexity of the latter type of images compared to images provided by other tools. In fact, under a mineralogical microscope, rock minerals exhibit both absorption colors, when observed in normal light, and birefringence colors when observed with crossed polarizers. Both observation modalities allow to acquire information for mineral identification and grain boundary detection. All of them are essential for mineral recognition and must be thoroughly evaluated to complete the thin section survey.

Some recent papers show how it is possible to address the problem of automatic segmentation of a thin section in transmitted light microscopy, combining the microscope with additive tools that allow a controlled rotation of the two polarizers (Feuten 1999; Goodchild and Feuten, 1998). However, when working at low magnification, mineralogical microscopes have a limited angle of view. Accordingly, only a limited portion of the thin section can be observed at once. In the case of coarse grained rocks, or when an image of the whole thin section is required, several images of the same sample have to be acquired, through an often complex image procurement strategy. The great improvement of the resolution of film color scanners (De Keyser, 1999), combined with their ability to scan a whole thin section in a single image, fully support their use in image acquisition and allows quantitative measurements.

We show in the following, a simple, cheap, and fast methodology for automatic grain-size analysis of standard thin sections whose images are acquired in 
transmitted light. This method, based on general purpose facilities, avoids the use of any dedicated costly scientific instrument. The image analysis procedure must be tuned for each set of sample. At present, it works satisfactorily for samples with relatively simple petrographic features. Of course this method is less general than the relatively complex ones described in the recent works mentioned above. Yet, it may represent for many petrologists a low cost alternative strategy providing an easy, and immediate access to new research themes.

\section{METHOD AND MEASUREMENTS}

\section{THE IN-HOUSE IMAGE ACQUISITION SYSTEM}

The entire analysis process is carried out with a low cost PC and a Nikon Coolscan II SCSI scanner (resolution up to $2700 \mathrm{dpi}$ ). The acquired images are stored as Tiff RGB files (1 byte-per color band), and processed using Noesis ${ }^{\mathrm{TM}}$ Visilog 5.2 macros. The image resolution can vary and is selected according to the grain size of the rocks under examination; the usual dimension of an image is $30 \times 20 \mathrm{~mm}$. The scanner is provided with a software allowing to chose optimal contrast, lightening and gain values.

An in-house mount has been devised to insert the thin section in the slot of the scanner between two removable polarizers. The acquisition of an image of a thin section just mimics the digitalization of a film slide. The mount is quite similar to a slide frame, with the exception that one side is open (U-shaped) to insert (resp. to extract) the section and the filters (Fig. 1). The filters may be removed or substituted independently (without removing the section or the mount from the scanner slot), allowing the acquisition of a series of perfectly collimated images of a same sample in different observation (or lighting) conditions.

When filters are not inserted in the mount, the resulting scanned image corresponds to an observation in "natural light" (called NP hereafter) just as in ordinary petrographic analysis under a petrographic microscope. When two crossing polarizing filters are inserted in the mount, the scanned image corresponds to a "crossed polarizers" observation (called NX hereafter).
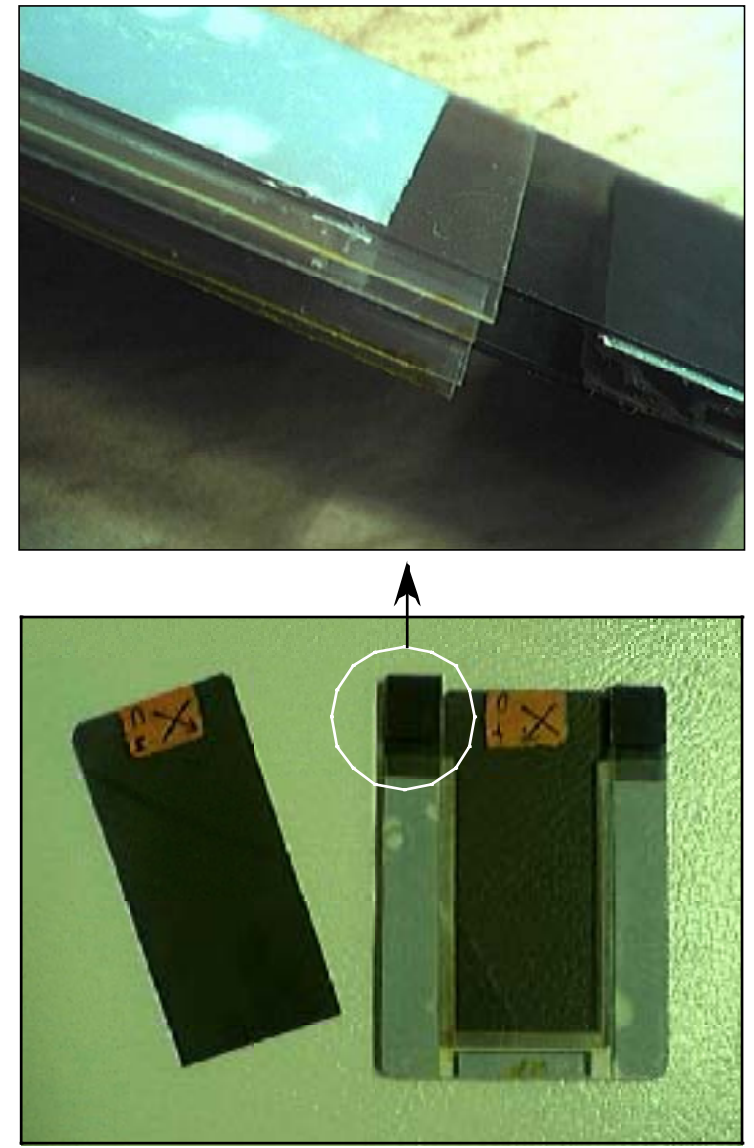

Fig. 1. Bottom: in-house device $(49 \times 68 \mathrm{~mm})$ prepared to insert the petrographic thin sections in the scanner. Top: detail showing the guides allowing the positioning of both sample and filters. Deep green slides are polaroids marked with the directions of polarization.

Several pairs of filters with orthogonal directions of polarization were cut from a common industrial polarizing gel sheet (linear Polarizer Polaroid HN38, $0.25 \mathrm{~mm}$ ). Polaroid pairs are cut with different orthogonal directions, allowing a variety of orientations. Thus, changing the couples of polarizers corresponds to rotating the stage of a polarizing microscope. No rotation can affect the thin section (in respect to the image acquisition system), allowing a perfect overlapping of a set of images of a same rock with different birefringence colors. Images acquired under crossed polarizers are used exclusively to detect mineral domains with different orientations, providing information on grain boundaries. In this respect, there is no need to carefully check orthogonality between the two polarizers. 


\section{METHODS OF GRAIN DETERMINATION}

The grain size analysis implies the distinction of each crystal. A crystal can be bounded by other crystals (or grains) belonging to the same phase or to different phases. Thus, both intra- and inter-phase boundaries need to be detected. Determining a rock grain size distribution- requires to identify all crystal boundaries and to assign a proper mineral phase to each crystal. In our examples, detection of grain boundaries is made using information derived from birefringence colors, while phases are detected on the basis of the differences in their absorption color. As a matter of fact, we consider here rocks whose minerals have a large birefractive power (olivine, pyroxenes, calcite). Even small changes in the orientation of the crystal lattice result accordingly in dramatic changes in birefringence colors. Rocks with such a property facilitate a complete grain orientation and modal analysis. Yet, our procedure may be used to identify mineral grain boundaries in other types of rocks with a less important birefractive power, simply by acquiring a larger number of cross-polarized images.

\section{THE ANALYZED SAMPLES}

Analyzed samples are (i) thin sections $(30 \mu \mathrm{m})$ from a wide series of ultramafic xenoliths from the upper mantle (harzburgites), and (ii) thin and ultra thin sections $(2-5 \mu \mathrm{m})$ from Carrara marbles. For clarity, the analysis is described hereafter separately for the two cases requiring different "tunings" of the algorithm.

i) The modal composition of peridotites is dominated by Olivine (influencing the mantle rheological properties) whose Crystal Size Distributions (CSD) is here to be obtained as a test for the method. The samples analyzed are fresh, spinel-bearing harzburgites from three suites of mantle xenoliths found in alkaline basalts from Canary Islands (Africa), Victoria Land (Antarctica) and Pali Aike (South America) (Perinelli, 2000). According to the classification proposed by Harte (1977), and Frey and Printz (1978), the rock textures vary from coarse and mosaic-equant to porphyroclastic. Olivine is Forich (Fo 89-92), colorless, and its modal abundance is between $60 \%$ and $85 \%$. Larger olivine crystals (up to $27 \mathrm{~mm}$ ) exhibit evident "kink-banding" and a preferred orientation Brownish enstatite (En 83-97, the dominant pyroxene) and very small amounts of green interstitial diopside (Wo 44.4-48.2, En 45.6-52, Fs 3.6-6.2), seldom reach a size larger than $5 \mathrm{~mm}$. Dark brown to reddish spinel is a minor interstitial component. The difference in absorption color between pyroxenes, spinel and olivine allows a straightforward mineral identification, and permits a fast modal analysis based on measurements of the area fraction of the minerals in the section. The scanner allows a resolution up to 2700 dpi (corresponding to 9.4 $\mu \mathrm{m} /$ pixel in our acquisition configuration). Due to the rock coarse grain size, the data set has been acquired here with a scanner working resolution of $1500 \mathrm{dpi}(\approx 17 \mu \mathrm{m} /$ pixel). However, to avoid to consider particles smaller than the section thickness $(\approx 30 \mu \mathrm{m})$, and to exclude any noise arising when considering as "grains" clusters of a few pixels, a filter has been adopted cutting particles smaller than $50 \mu \mathrm{m}$.

Prior to interpreting CSD data, one shall evaluate the effects on the CSD of left hand side and righthand side truncations effects (Pickering et al.,1995). Our device allows to determine harzburgite CSD data in the range $0.15 \mathrm{~mm}$ to $20 \mathrm{~mm}$. Left-hand-side truncation effects yield a decrease of the grain density number. They appear here below $0.15 \mathrm{~mm}$ as a consequence of the working resolution of $1500 \mathrm{dpi}$ and can be limited using a higher magnification (Armienti et al.,1994).

Right-hand-side truncation effects (due to undersampling) flatten size distributions at large grain size, resulting in a constant value of the number density for classes that contain at most one particle. Right-hand-side truncation effects can be limited by enlarging the analyzed area (e.g. by using up to ten sections for each mantle xenolith sample) or adopting a logarithmic variation for the width of the class size. The minimum area that has to be examined to avoid right-hand-side truncation can be evaluated by means of the relation (Armienti et al., 1994):

$$
\mathrm{A}_{\text {min }}=\frac{1}{2 \mathrm{n}(\mathrm{L}) \cdot \Delta \mathrm{L} \cdot \sqrt{\mathrm{L} \cdot \Delta \mathrm{L}}},
$$

where $\mathrm{n}(\mathrm{L})$ is the measured number density, $\Delta \mathrm{L}$ is the adopted size interval and $\mathrm{L}$ is the grain size. 


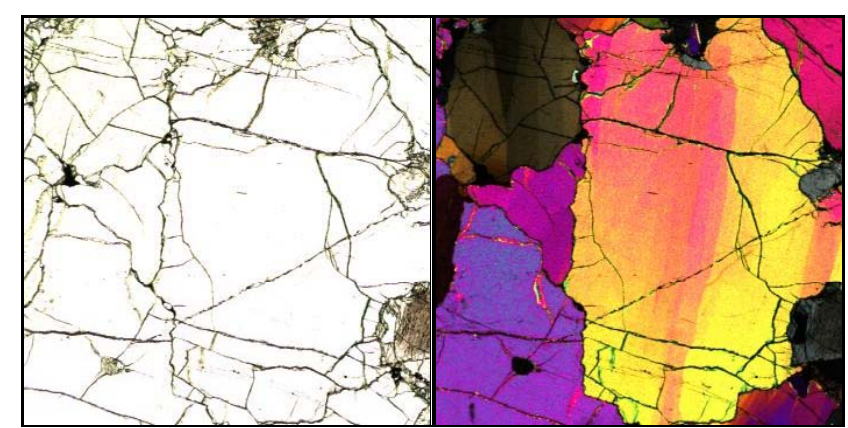

a)

b)

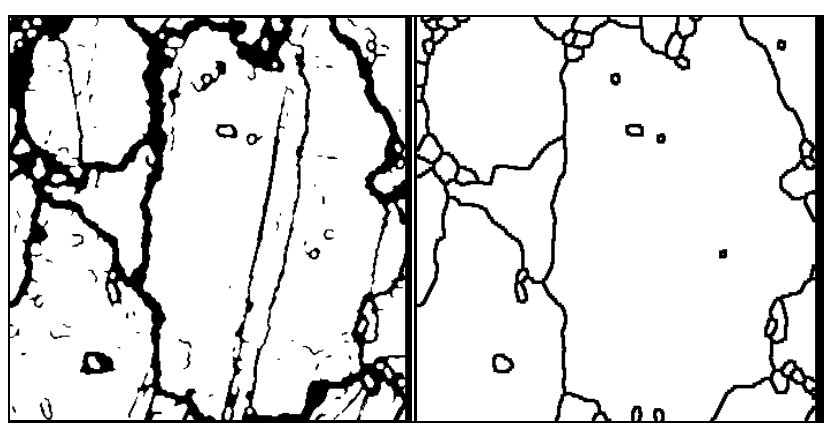

c)

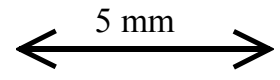

d)

Fig. 2. a) NP image, b) NX image, c) threshold gradients, d) result image (from 4 NX images). Small individuals inside large crystals represent artefacts generated by intra-crystalline micro-fractures; filtering may eliminates this noise.

In an image such as Fig. 2a, acquired in parallel light (NP), discontinuity elements are represented by Crystal Boundaries (CB), mineral fractures, and cleavages.

To find and properly trace only the $\mathrm{CB}$, it is possible to look for birefringence color gradients in each NX image applying the command "compass $3 \times 3$, Prewit 1" of the Visilog package. Marginal gradients are thus obtained for each RGB channel of each (differently oriented) NX image. A suitable threshold of the steepest gradient values in each band produces a set of binarized R-gradients, G-gradients, and B-gradients whose union over the whole series of NX images provides the information about the candidate $\mathrm{CB}$ in a binary image (hexagonal grid) (Fig. 2c). Yet, it is clear that in an NX image (e.g. Fig. 2b) the weaker gradient may not refer to true CB. This concerns kink-banding within olivine crystals in which slight distortions of lattice orientation produce nuances in birefringence. To avoid this ambiguity, a control is introduced on the steepness and continuity of each gradient trace, by tuning the threshold value and discarding this way discontinuous and weaker gradients (Figs. 2c-d).
Also, not all NX images show a gradient across a CB due to analogous birefringence color of two or more adjacent crystals in a particular polarizer orientation, or due to effective iso-orientation of these crystals). Generally the latter case is rare, but may require additional CB detectors when widely encoutered. Practically, acquiring 4 NX images with different polarizer orientations turns out to be sufficient to make all gradients appear. Note that individual crystals are identified within a noise (Fig. 2c) eliminated relying on the Visilog "skiz" algorithm (Figs. 2c-d). The resulting image (e.g. Fig. 2d) commonly outlines each crystal boundary. In NP images, the simple mineralogical composition of the samples permits the identification of the three principal components: olivine, pyroxenes and spinel. The principle is based on that olivine crystals are colorless, pyroxenes are usually brownish and darker than olivine, and spinel appears in dark brown. The markers for all three phases are separated by simple thresholds on the G channel of the original RGB NP image (the most appropriate due to the brownish absorption colors). Markers are then combined with CBs to assign to each grain its mineral phase. At this point, modal and grain size analysis is completed, and morphometric parameter can be measured for each phase.

ii) Ultra thin (Cantisani et al., 2000) and standard sections of white Carrara marbles are the second set of analyzed samples. These marbles are practically pure carbonate $\left(\mathrm{CaCO}_{3}>99 \mathrm{Wt} \%\right.$; Molli et al., 1999), and show a statically recrystallised microfabric with a granoblastic structure and polygonal grain boundaries (mean size is about $200 \mu \mathrm{m}$ ). They belong to a structural domain of the Alpi Apuane where relatively coarse microfabrics developed upon annealing induced by recrystallization. The process develops at relatively high temperature metamorphic regime $\left(\mathrm{T} \approx 360-430^{\circ} \mathrm{C}\right)$. These samples were processed using the maximum scanner resolution (2700 dpi) with grains smaller than 5-7 pixels remaining below the resolution limit of the procedure. Calcite being the only observed phase, only CB need to be detected. The detection of CB described above works very well in these marbles, and textures are satisfactorily reconstructed. However, slightly different procedures are needed depending on whether ultra thin or standard sections are considered.

On the one hand, ultra thin sections are so well polished that NX images provide a very clean information towards a good texture determination 
(Fig. 3a). The main difficulty in analyzing marbles based on birefringence gradients is actually to avoid separation of grains induced by calcite twinning. An initial opening-closing filter in the input RGB NX image drastically limits this problem shrinking and canceling the narrow tracks of twinning (Fig. 3).

On the other hand, standard marble thin sections yield to NX images which are less accurate and more noisy. In this respect, additional $\mathrm{CB}$ information must be extracted from NP images. The NP image is converted from RGB to HLS. The L image component is then thresholded to separate dark tracks (lowest lightness values) assumed to represent essentially $\mathrm{CBs}$ as no microfracturation affects calcite crystals in our samples.

\section{THE STEREOLOGICAL REGRESSION OF 2-D DATA}

Particle size measurements (the diameter of the circle of equivalent area) are obtained from the binary images of grain boundaries and converted in 3-D size distribution by correcting data by means of the Schwartz-Saltikov method (DeHoff and Rhines, 1972; Armienti et al., 1994; Higgins, 2000). This allows the calculation of the Crystal Size Distributions (CSD) expressed as the crystal number density N(L) $v s$ size (L). Both linear and logarithmic scaling of class sizes have been performed to optimize statistics for the largest grains (less abundant) and to reveal the details of the distribution of the smallest grains. Data are plotted as $\log \mathrm{N}(\mathrm{L})$ vs $\log (\mathrm{L})$ diagrams (Figs. 4 and 5). Evaluation of left-hand-side (due to resolution) and right-hand-side truncation (due to sample size) of the CSD is performed according to Pickering et al. (1995). In Fig. 4d and 5c, the left-hand-side truncation appears as a sudden drop in the number of small crystals. Right-hand-side truncation for the largest crystals of harzburgites always appears as an increase of the slope of the CSD and may be less (Fig. 4d) or more evident (Fig. 4e) depending on the sample. Right-hand-side truncation effects may be reduced by calculating the CSD of several sections of a same sample (more details in Armienti and Tarquini, 2002). Note in Fig. 4d the smoothing effect of the logarithmic binning revealing a linear correlation.

For all ultramafic xenoliths, a linear relation log $\mathrm{N}(\mathrm{L}) v s \log (\mathrm{L})$ diagrams is evidenced, supporting the olivine CSD scale invariance over sizes ranging from about 0.2 up to more than $10 \mathrm{~mm}$. The slope of the CSD in a Log-Log diagram corresponds to the fractal dimension of the distribution (Simakin et al., 1999; Armienti and Tarquini, 2002). The observed CSD fractal dimension varies from 2.5 to 3.5. Such values are in agreement with distributions of clast sizes found in a variety of geological contexts (Turcotte, 1995) and are explained by fragmentation mechanisms consistently with the porphyroclastic texture recognized in the analyzed ultramafic xenoliths. Mosaic equant marbles exhibit a markedly different size distribution, with no scale invariance (Fig. 5). The distinct size distribution patterns can be related to the different mechanisms of genesis of this rock, involving annealing of grains during static recrystallization occurring during thermal peak events (Molli et al., 1999).

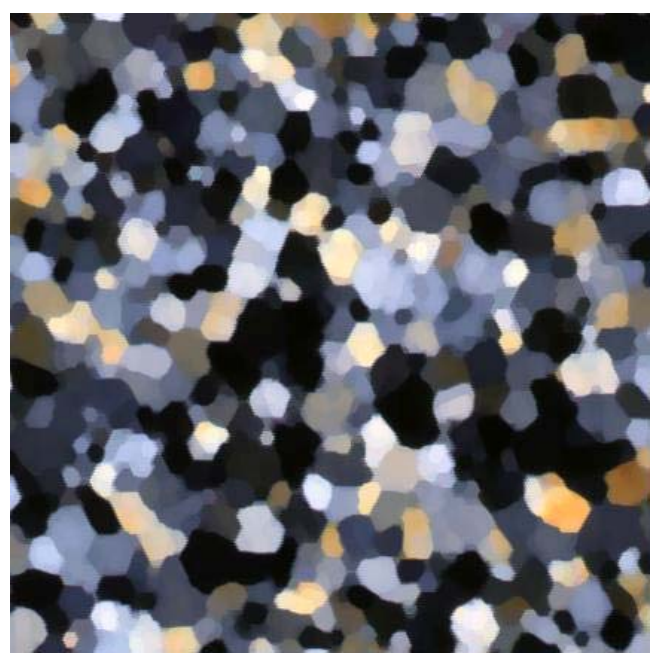

b)

Fig. 3. Ultra-thin (5 $\mu \mathrm{m})$ section of Carrara marble; 3a) Original NX image with evident traces of twinning and cleavages within the crystals. Long scratches across the image are also evident. 3b) Twinning, cleavages and scratches of calcite crystals are removed after four iterations of an opening-closing filter ( $4^{\text {th }}$ order). 

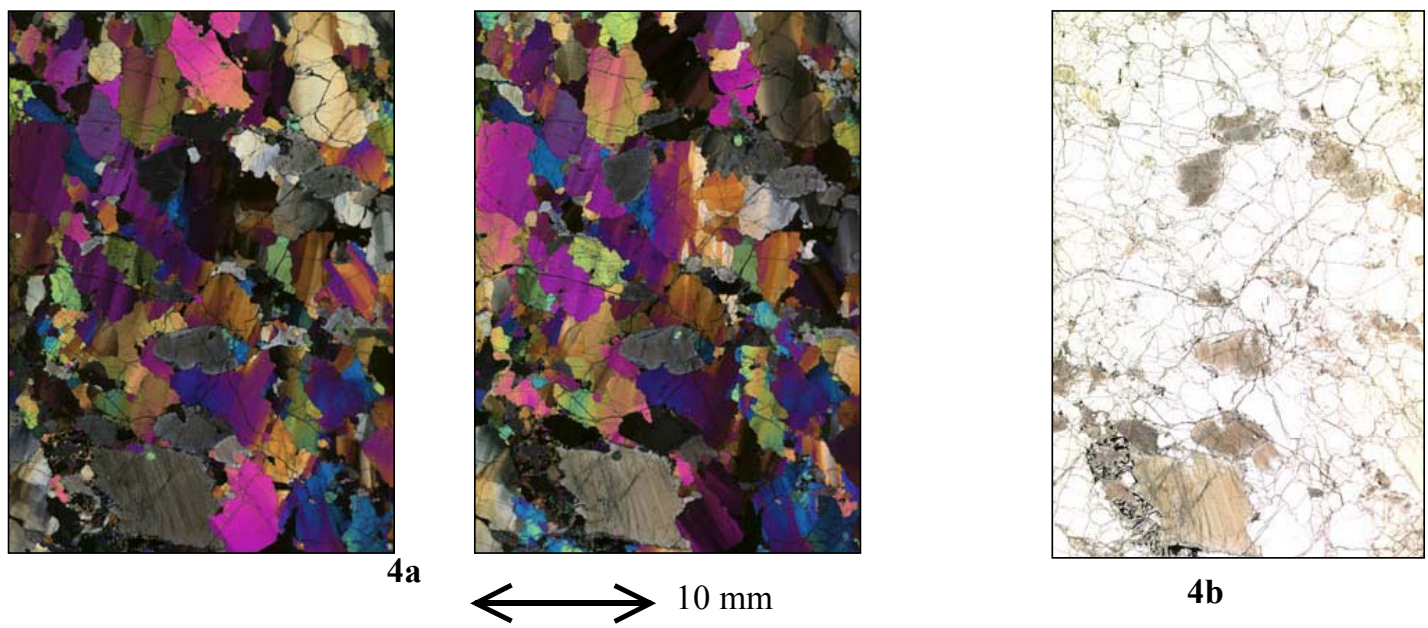

4b
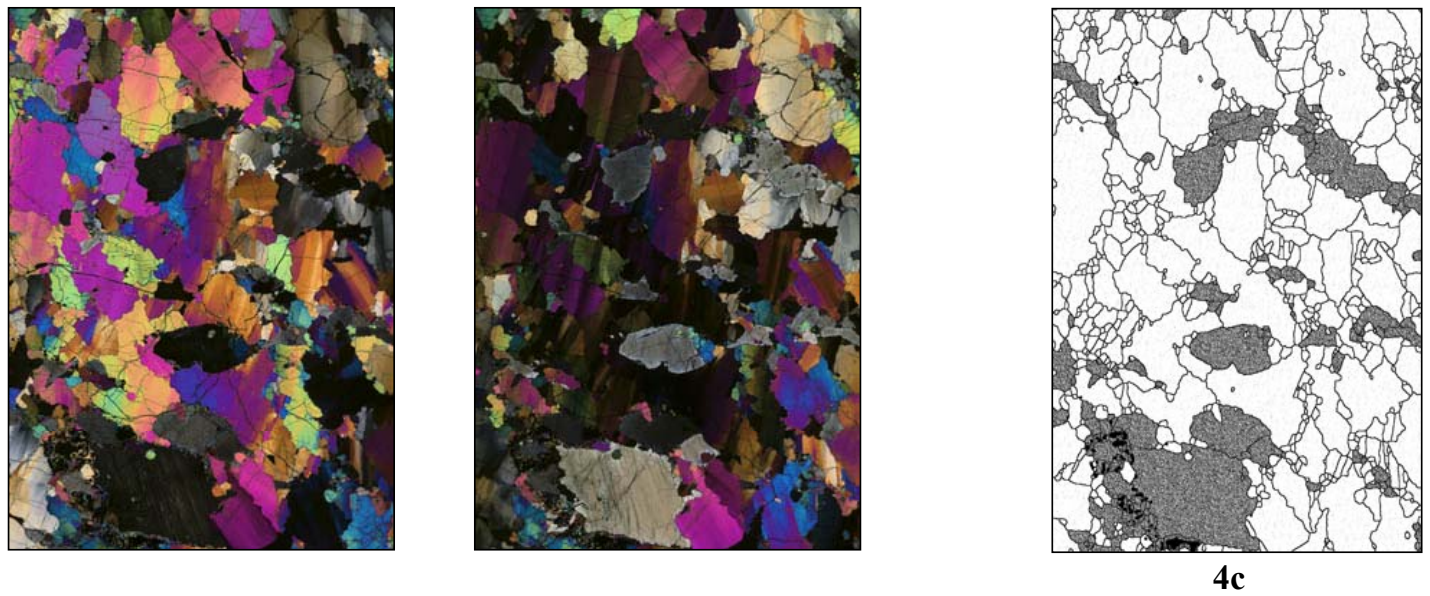

Fig. 4a-c. Input and output images for the analysis of an harzburgite thin section, $(22.6 \times 32.3 \mathrm{~mm}) .4 \mathrm{a}:$ the four NX input images; $4 b$ : the single NP input images; $4 c$ : the output image with olivine grains in white, pyroxene in gray and spinel in black.

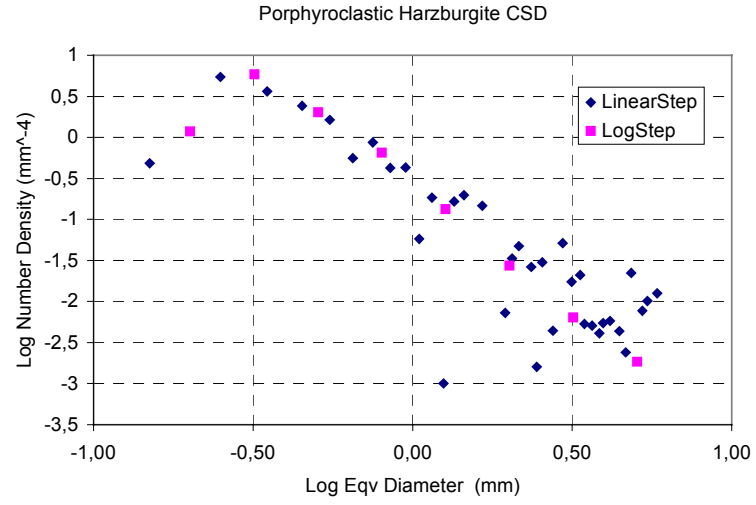

4d)

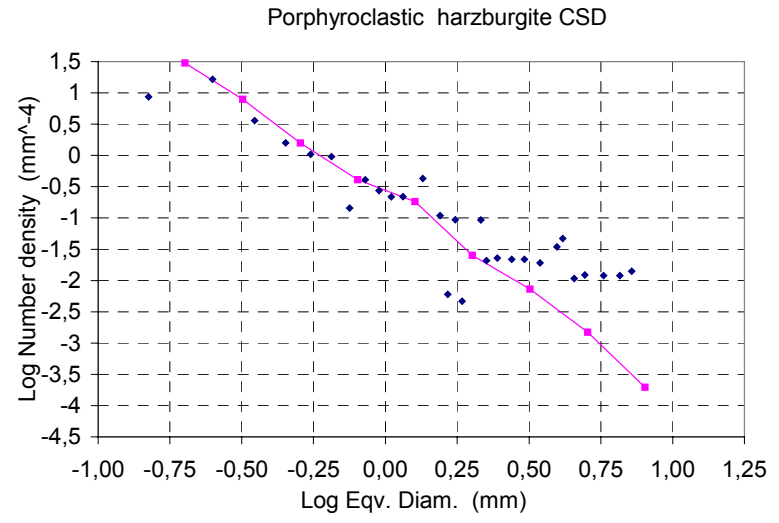

$4 \mathrm{e})$

Fig. 4d-e. 4d: Olivine CSD of 4c after stereological correction. 4e: Olivine CSD of another sample of the suite. The right-hand-side truncation in linearly binned size classes may appear at sizes larger than $2 \mathrm{~mm}$. Note that the right-hand-side truncation is less evident in $4 d$ and is more pronounced in $4 e$. In these thin sections the analyzed area is about $600 \mathrm{~mm}^{2}$ and $\Delta L$ is $0.1 \mathrm{~mm}$ for linearly binned size intervals. 

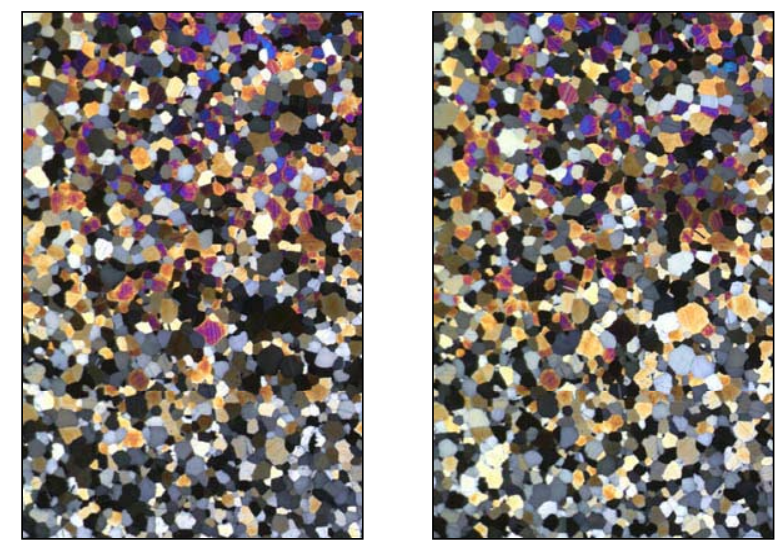

$5 \mathbf{a}$
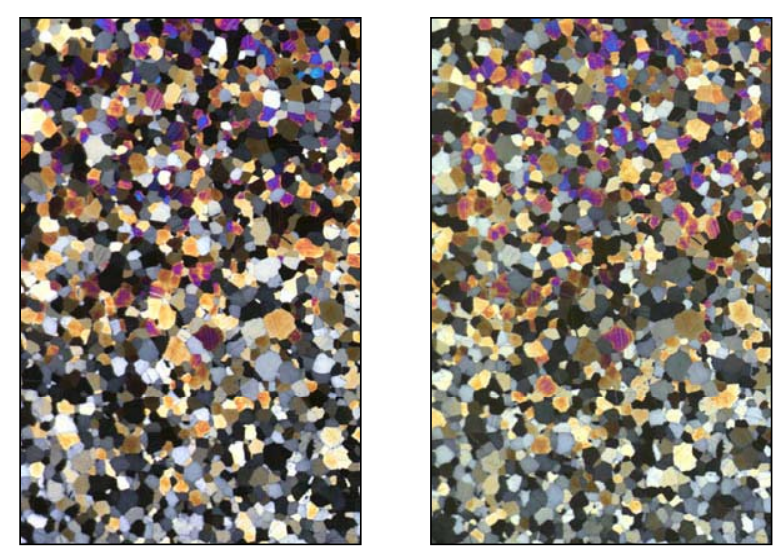

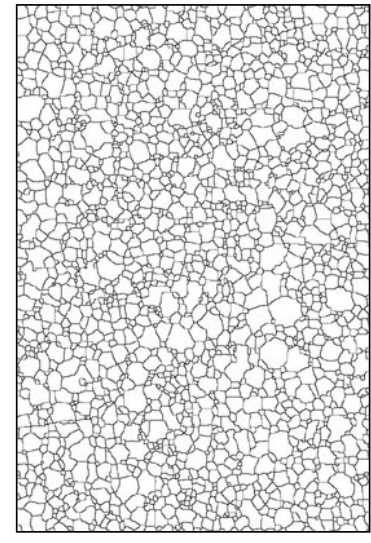

$5 \mathbf{b}$

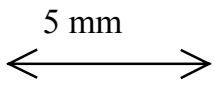

Fig. 5a-b. The input and output images for the analysis of a marble ultra thin section, $(8.2 \times 12.3 \mathrm{~mm})$. $5 \mathrm{a}$ : the four NX input images; $5 b$ : the output image with determined texture.

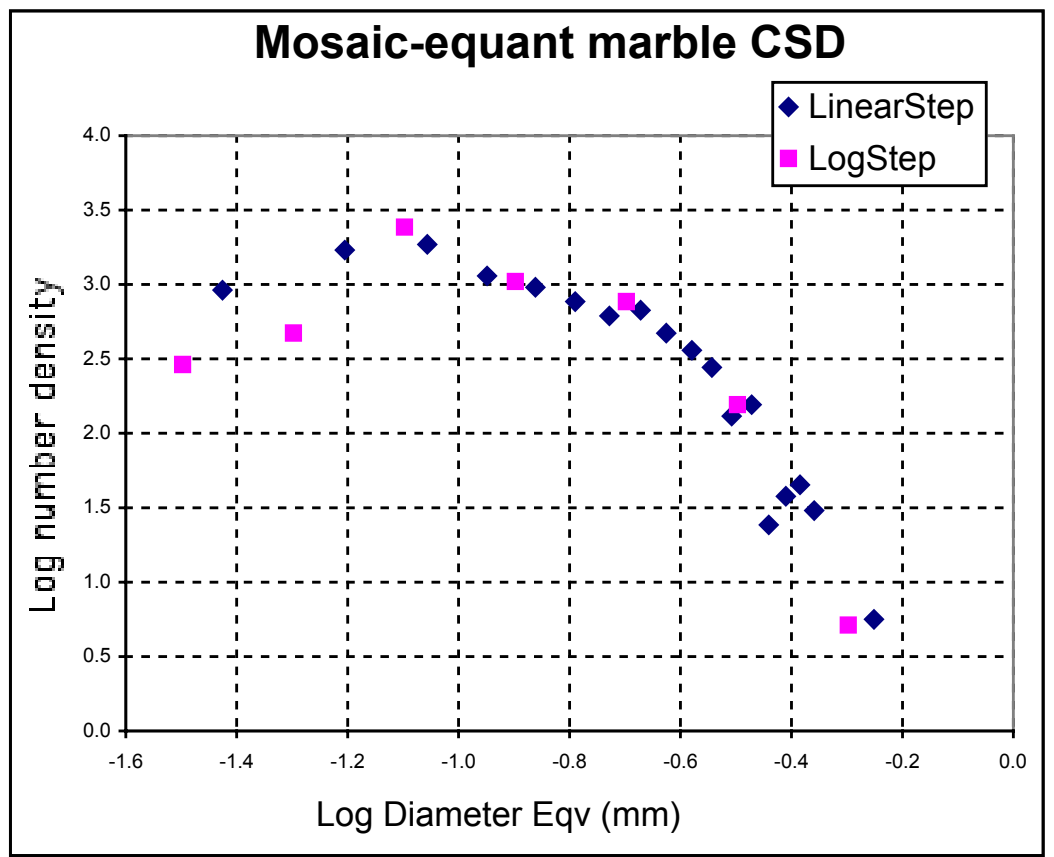

Fig. 5c. CSD of $5 b$ after stereological correction. 


\section{CONCLUSIONS}

Combining general purpose facilities with image analysis tools, grain size distributions from thin sections of harzburgites and marbles have been determined. The observed CSD patterns are consistent with the petrological processes that drive rock genesis.

The designed method allows to quickly obtain accurate grain size distribution measurement at low cost. In the case of mantle xenoliths the achieved results are in agreement with fragmentation processes, as discussed by Turcotte (1995).

It is to be noted that any variation in the sample features (occurrence of alteration, different section thickness and so on) requires a tuning session including the calibration of parameters or the inclusion of additional procedure blocks (e.g., additive $\mathrm{CB}$ detection from NP). Moreover, the imaging device itself has a fixed higher resolution limit $(1 \mathrm{pixel}=9.4 \mu \mathrm{m})$ hampering any further enhancement of measurements. This is also a limit for the analysis of very fine grained rocks. The image analysis procedure may be exported to process series of images acquired with other imaging devices like CCD cameras and microscopes. It should be also underlined that, in transmitted light, the size of the smallest detectable crystals must be larger than the section thickness (usually $30 \mu \mathrm{m}$ ). This avoids to assume erroneously that crystals contained in the section volume intersect the section surface, as required by the Schwartz-Saltikov procedure. In rocks with a very coarse grain size, even if $\mathrm{CB}$ are detected, CSD may be subject to heavy effects of right-hand truncation. In these cases more than a section is to be used to collect reliable data. A further limit concerns the recognition of the different phases based on a simple evaluation of absorption color implying that rock phases with similar colors cannot be completely differentiated.

\section{ACKNOLWEDGMENT}

This work was founded by the grant MURST 1998 N. 9804307319_002: "Materiali terrestri ed analoghi sintetici ad alta pressione ed alta temperatura: proprieta' fisiche, chimiche e reologiche". The authors wish to thank Y. Anguy for his suggestions that substantially improved this paper.

\section{REFERENCES}

Armienti P, Tarquini S (2002). Power-law olivine cystal size distributions in lithospheric mantle xenoliths. Lithos 65:273-85
Armienti P, Pareschi MT, Innocenti F, Pompilio M (1994). Effects of magma storage and ascent on the kinetics of crystal growth. The case of 1991-93 Mt. Etna eruption. Contrib Mineral Petrol 115:402-14.

Cantisani E, Canova R, Fratini F, Manganelli Del Fa C, Mazzuoli R, Molli G (2000). Relationship between microstructures and physical properties of white Apuan marbles: inferences on weathering durability. Period Mineral 69(3):257-68.

De Hoff RT, Rhines FN (1972). Microscopie Quantitative. Paris: Masson et Cie eds, 404.

De Keyser TL (1999) Digital Scanning of thin sections and peels. Research method papers. J Sediment Res 69(4):962-4.

Feuten F (1999). Petrographic image processing of thin section using the rotating polarizer stage. Geovision 99: Proceedings of the International Symposium on Imaging Application in Geology. Univ of Liège, Belgium, 105-8.

Goodchild JS, Feuten F (1998). Edge detection in petrographic images using the rotating polarizer stage. Comput Geosci 24:745-51.

Frey FA, Prinz M (1978). Ultramafic inclusion from San Carlos, Arizona: petrologic and geochemical data bearing on their petrogenesis. Earth Plan Sci Lett $38: 129-76$.

Harte B (1977). Rock nomenclature with particular relation to deformation and recrystallization texture in olivine-bearing xenoliths. J Geol 85:279-88.

Higgins MJ (2000). Measurement of crystal size distributions. Americ Mineral 85:1105-16.

Molli G, Conti P, Giorgetti G, Meccheri M, Oesterling N (1999). Microfabric study on the deformational and thermal history of the Alpi Apuane marbles (Carrara marbles), Italy. J Struct Geol 22:1809-25.

Perinelli C (2000). Modificazioni indotte dal metasomatismo di alta pressione ed alta temperatura nelle peridotiti del mantello superiore in zone di rift attivo, di margine passivo e di magmatismo d'intraplacca., Ph. D. thesis, XII ciclo. Università di Pisa, 98 pp.

Petruck W (1988). The capabilities of the Microprobe image analysis system: application to mineral beneficiation. Scanning Microsc 2(3):1247-56.

Pickering G, Bull JM, Sanderson DJ (1995). Sampling Power-law distributions. Tectonophysics 248:1-20.

Simakin AG, Armienti P, Epel'baum MB (1999). Coupled degassing and crystallization: experimental study in continuous pressure drop, with application to volcanic bombs. Bull Volcanol 61:275-87.

Turcotte DL (1995). Fractals and chaos in geology and Geophysics. Cambridge: Cambridge University Press, $221 \mathrm{pp}$. 